\title{
What is the correct staging and treatment strategy for locally advanced prostate cancer extending to the bladder?
}

\author{
Özgür Haki Yüksel, Ayhan Verit, Ahmet Ürkmez \\ Fatih Sultan Mehmet Research \& Training Hospital, Dept. of Urology, Istanbul, Turkey
}

\begin{abstract}
Summary In locally advanced prostate cancer with bladder invasion, frequently encountered problems such as bleeding, urinary retention, hydronephrosis, and pain create distress for the patients. Therefore patients' quality of life is disrupted and duration of hospitalization is prolonged. Relevant literature about accurate staging and treatment of locally advanced prostate cancer with bladder invasion was investigated.

Locally advanced prostate cancer can present as a large-volume aggressive tumor extending beyond boundaries of prostate gland, and involving neighboring structures which can be involved as recurrence(s) following initial local therapy. Survival times of these patients can range between 5 and 8 years. Their common characteristics are adverse and severe local symptoms unfavorably affecting quality of life Control of local symptoms and their effective palliation are independent clinical targets influencing survival outcomes of these patients.

The treatment outcomes of locally advanced prostate cancer into the bladder are currently debatable. Although in the current TNM classification, it is defined in T4a, we think that this may be categorized as a subgroup of $\mathrm{T} 3$ and thus encourage surgeons for the indication of radical surgeries (radical prostatectomy, radical cystoprostatectomy) in selected patient populations after discussing issues concerning consequences of the treatment alternatives, and expectations with the patients. Cystoprostatectomy followed by immediate androgen deprivation therapy may be a feasible option for selected patients with previously untreated prostate cancer involving the bladder neck because of excellent local control and long term survival.
\end{abstract}

KEY WORDS: Bladder neck invasion; Cystoprostatectomy; Locally advanced prostate cancer; Radical prostatectomy; Staging.

Submitted 3 November 2014; Accepted 31 December 2014

\section{INTRODUCTION}

Locally advanced prostate cancer (LAPC) has been clinically described as a cancer extending beyond prostate capsule, with invasion of pericapsullar tissue, apical region of the bladder, bladder neck or seminal vesicles without lymph node involvement or distant metastases. In clinical staging, they are categorized as T3-T4 NO MO prostate cancer (PC) and constitute $10-20 \%$ of the cases with newly diagnosed cases of PC (1). While in previous reports, nearly $40 \%$ of the cases with newly diagnosed PC were categorized in clinical stage T3 (cT3), currently its incidence ranges between 5-15\% (2). Approximately $5-15 \%$ of the PC cases are located in clinical stage of T4. In current TNM staging, bladder neck invasion (BNI) has been categorized as stage T4 disease (Table 1).

Management of localized PC and metastatic disease has been summarized in algorithms, although optimal treatment of clinical stage T3-T4NOMO PC is intensively debated. One of the main reasons of these debates is related to inaccurate local clinical staging over T2 or under staging of positive lymph node (LN) (3).

According to European Association of Urology (EAU) guidelines, watchful-waiting, radiotherapy (RT), radical retropubic prostatectomy (RRP), androgen deprivation therapy (ADT) and various combinations of these treatment modalities based on general clinical condition of the patient and local invasion of the tumor can be used (Table 2). According to the current scientific data, multimodality therapy was recommended for the majority of patients. Treatment of locally advanced disease aims at two fundamental goals; the first goal is complete elimination of the disease and the second one is achievement of local control. In terms of biological behavior of PC, low, medium and high risk groups are defined. For high-risk disease, 'clinical T3', 'locally advanced', 'bad differentiated' terms are used and however these are not sufficient to identify the disease (4). Definition of high-risk PC is associated with different criteria according to different sources as summarized in Table 3.

D'Amico risk classification grouping is the most widely used. Accordingly, prostate-specific antigen (PSA) $\geq 20$ $\mathrm{ng} / \mathrm{mL}$ or Gleason score (GS) 8-10 or clinical stage $\geq \mathrm{cT} 2$ are considered as high-risk criteria of prostate cancer (5). American Urological Association (AUA) has also used this risk stratification since 2007 (6). Traditionally, urologists prefer RT and ADT rather than RRP for the treatment of high risk PC. Population-based Surveillance, Epidemiology and End Results (SEER) cancer data examination results showed us that in clinical T3 disease the rate of patients who underwent RRP, decreases from 18.1 to $9.1 \%$ and RT rates increase of $20 \%$ (7). In patients under the age of 70 who were diagnosed with high-risk prostate cancer, $25 \%$ of them could not receive an effective local treatment (2). According to the Cancer of the Prostate Strategic Urological Research Endeavor (CaPSURE) data, most of the newly diagnosed patients with highrisk localized PC canalize to the $\mathrm{ADT}$ as if the unique 
Table 1.

2009 TNM staging system of the PC.

\section{T: Primary tumor}

Tis: No evidence of primary tumor

T1a: Tumor detected in less than $5 \%$ of the total TUR [transurethral prostate resection] material, normal DRE findings, total Gleason score $<7$ pts

T1b: Tumor detected in more than $5 \%$ of the total TUR material, normal DRE, Gleason score $>7 \mathrm{pts}$

T1c: Tumor diagnosed based on the results of the fine needle biopsies performed because of increased PSA levels in a group of patients with normal DRE estimates

T2a: Tumor involving only half or less than $50 \%$ of one lobe of the prostate

T2b: Tumor involving more than one half of one lobe

T2c: Tumor involving both lobes

T3a: Uni or bilateral extra-capsular invasion

T3b: Seminal vesicular involvement

T4a: Involvement of bladder neck, external sphincter or rectum

T4b: Tumor invading pelvic wall

\section{$\mathrm{N}$ : Regional lymph nodes}

NO: No evidence of invasion

N1: A single metastatic lymph node measuring $2 \mathrm{~cm}$ in diameter

N2: A single metastatic lymph node measuring $2-5 \mathrm{~cm}$ or multiple metastatic lymph nodes each measuring $<5 \mathrm{~cm}$ in diameter

N3: A metastatic lymph node larger than $5 \mathrm{~cm}$ in diameter

\section{M: Distant metastases}

MO: No evidence of metastasis

M1a: Metastasis to non-regional lymph nodes

M1b: Bone metastasis

M1c: Distant rgan metastasis

\section{Table 2.}

2013 EAU guidelines in the light of the treatment of locally advanced PC, some of the alternatives.

\begin{tabular}{|c|c|c|}
\hline Mode of treatment & Indications and general information & Degree of evidence \\
\hline Watchful waiting & $\begin{array}{l}\text { Asymptomatic, well and moderately differentiated tumors and patients with low life expectancy } \\
\text { and a life expectancy } \leq 10 \text { years }\end{array}$ & 3 \\
\hline \multirow[t]{5}{*}{ Radical prostatectomy } & Selected cT3a, Gleason $\leq 8$, PSA $\leq 20 \mathrm{ng} \mathrm{mL}$ and life expectancy $\geq 10$ years & 3 \\
\hline & Selected cT3b-T4, N0 or someone T and N1: Multimodality practicable treatment & 3 \\
\hline & T3a: Unilateral nerve-sparing surgery & 4 \\
\hline & RP+Adj HT [Bicalutamide 150 mg1x1] useful in increasing progression-free survival & \\
\hline & NHT+RP: ineffective overall survival or disease-free survival & \\
\hline \multirow[t]{5}{*}{ Radiotherapy } & $\begin{array}{l}\text { T3NoMo In the early postoperative period in patients with adjuvant RT [positive surgical margins, } \\
\text { especially in the] progression-free survival helpful }\end{array}$ & 1 \\
\hline & $\begin{array}{l}\text { T2-T3NoMo, in patients with persistent elevated PSA recurrence of elevated PSA and PSA } 0.5 \mathrm{ng} \mathrm{ml} \\
\text { before RT can be given in recovery }\end{array}$ & 3 \\
\hline & $\begin{array}{l}\text { T3-T4NoMo ve WHO performance status-2 for patients; concomitant and adjuvant hormone therapy [ } 3 \mathrm{yr} \text { ] } \\
\text { is beneficial to overall survival }\end{array}$ & 1 \\
\hline & T2c-T3 No-x, Gleason 2-6 in patients neo-adjuvant and concurrent with short term ADT overall survival was helpful & $1 b$ \\
\hline & $\begin{array}{l}\text { High-risk N1Mo and without severe comorbidities in patients pelvic external beam RT and concurrent } \\
\text { long-term adjuvant hormone therapy, overall survival, biochemical control is useful, due to disease } \\
\text { and the development of metastases associated with failure delays }\end{array}$ & $2 b$ \\
\hline \multirow[t]{3}{*}{ Hormonotherapy } & Symptomatic, T3-T4, PSA > 25-50 ng mL, PSA doubling time $<1$ year for patients & 1 \\
\hline & Patients suitable for RT are not suitable for hormonal monotherapy & \\
\hline & Nonsteroidal antiandrogen monotherapy is an alternative to castration & $2 a$ \\
\hline
\end{tabular}

Table 3.

Definitions of high-risk prostate cancer

\begin{tabular}{|c|c|}
\hline Source & Definition \\
\hline 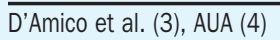 & PSA $\geq 20 \mathrm{ng} / \mathrm{ml}$ or GS $8-10$ or Clinical Stage $\geq \mathrm{T} 2 \mathrm{c}$ \\
\hline EAU (5) & PSA $\geq 20 \mathrm{ng} / \mathrm{ml}$ or biopsy $\mathrm{G} 8-10$ or Clinical stage $\geq \mathrm{T} 3 \mathrm{a}$ \\
\hline RTOG (6) & PSA 20-100 ng/ml, biopsy GS 8-10 and Any clinical stage or clinical stage $\geq \mathrm{T} 2 \mathrm{c}$ or PSA $<100 \mathrm{ng} / \mathrm{ml}$ and GS8-10 \\
\hline NCCN (7) & PSA $>20 \mathrm{ng} / \mathrm{ml}$ or GS $8-10$ or clinical stage $\geq \mathrm{T} 3$ or $\mathrm{T} 2 \mathrm{~b} / \mathrm{c}, \mathrm{GS}=7, \mathrm{PSA}>10$ any two of the parameters \\
\hline Eastham et al. (8) & Kattan nomograms in the 5-year progression probability $\leq 50 \%$ \\
\hline 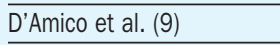 & Preoperative PSA velocity > year $2 \mathrm{ng} / \mathrm{ml}$ \\
\hline
\end{tabular}


valid treatment option (8). Although in the current TNM classification it is defined in clinical stage of $\mathrm{T} 4 \mathrm{a}$, we think that LAPC invading the bladder may be categorized as a subgroup of cT3 in order to encourage surgeons to the indication of radical surgeries (RRP, cystoprostatectomy) in selected patient populations and after discussing with the patients issues, concerning consequences of the treatment alternatives and expectations. Altogether, we think that clinicians should not strictly obey the classical staging systems in every cases, especially when LAPC invaded the bladder.

\section{Discussion}

In LAPC, RT and ADT are considered as standard treatment modalities, however in selected cases, RRP is recommended as a primary treatment alternative. An advantage of RRP which should not be overlooked is that RRP can demonstrate true histopathological stage of the primary cancer and evaluate potential LN invasion. By this modality, patients with disease recurrence and progression risks can be determined. In fact, patients with organ-confined disease (pT2) can be spared from morbidities of adjuvant treatment modalities.

Extracapsular invasion is found in $88-91 \%$ of the cases with clinical manifestations of LAPC, while surgical margin positivity (PSM) and seminal vesicle involvement (SVI) have been reported in $22-53 \%$ and $23-29 \%$ of the cases, respectively (9).

Whether BNI conveys a high risk for biochemical progression following RRP is a debatable issue. In TNM staging system, BNI is not defined in detail with respect to its microscopic and macroscopic characteristics.

Independent prognostic value of microscopic BNI in PC is not clear. In a study encompassing 1845 patients, conically resected bladder neck specimens were evaluated and true BNI was defined as PC focus within thick smooth muscle bundles without intermixed benign prostatic tissue. While false BNI was described as PC cells intermixed with benign prostatic tissue. BNI was evaluated and analyzed within the context of preoperative serum PSA levels, extra prostatic invasion, SVI, PSM, LN involvement, previous RRP (if any), GS and tumor volume in 90 of 1845 patients diagnosed with BNI. Sixtythree cases of 90 (4.9\% of 1845$)$ patients with microscopic BNI were classified as true BNI and 27 cases were categorized as false BNI. In patients with BNI, time to biochemical failure was similar in patients with negative and positive surgical margins (Kaplan-Meier curves). Even though BNI is related to other pathologic features, as demonstrated in previous studies, it is not an independent marker of PSA recurrence. In the light of previous and currently available data, the authors had expressed their expert opinions about TNM staging system and indicated that staging system should be reviewed with respect to BNI and these group of patients should be included in the clinical stage of T3a with favorable prognosis (10).

Dash et al. performed a univariate analysis on their 1123 cases with localized PC and estimated relative probabilities for PSA recurrence risks as 1.52, 3.05 and 8.59 for patients with BNI, extraprostatic tissue invasion and SVI, respectively (11).
In a study conducted by Ruano et al. in a series of 290 consecutive patients, 55 cases who presented with BNI and a subgroup of 18 cases also showing PSM according to microscopic criteria of Yossepowitch were compared to patients with extraprostatic extension or seminal vesicle invasion showing no statistically significant intergroup difference as assessed by Cox proportional hazard model. In conclusion, patients with microscopic BNI after RRP had higher preoperative PSA values and GS, higher PSM, advanced pathologic stage and larger sized tumors when compared to those with extraprostatic involvement but showed similar biochemically detected recurrence-free rates. Furthermore, their outcomes were more favorable in comparison to the patients with seminal vesicle involvement and the Authors indicated the need for inclusion of these tumors in clinical stage T3a (12).

Problems encountered in radical surgery performed for LAPC with BNI include higher rates of SVI and LN positivity when compared to the patients with localized disease. However some studies have demonstrated absence of any biochemically detected disease progression within 10 years after RRP in 25\% of the patients with SVI and without metastatic LN (13). As a reason for this outcome, discrepancies in the definition of SVI have been indicated (14).

Secin et al. evaluated 387 preoperatively treatment naive patients with SVI who had undergone RRP and published their 15-year follow-up outcomes (15). In this study, 10 and 15 year disease-free survival rates of 296 (76\%) patients with SVI but no LN involvement were reported as 89 and $81 \%$, respectively. However their biochemical recurrence rates were indicated as 64 and 68\%, respectively. In the same study, 10-year biochemical disease-free and disease-specific survival rates for 92 patients with SVI and LN involvement were reported respectively as 10 and $74 \%$. Nevertheless, at the end of 10 years, $66 \%$ of the patients were still surviving (7). Masterson et al. analyzed 24 patients with LN positivity and SVI and reported 5-year biochemical disease-free survival rate as $25.9 \%$ and they estimated mean time interval up to the PSA progression as 6 months (16). Boorjian indicated that in cases with only one LN involvement, the risk of disease-specific mortality had increased 4-fold which was two times higher in patients with multiple LN involvement (17).

In a higher-stage LAPC, as supported by the outcomes of some literature studies, extended LN dissection can be recommended instead of standard pelvic LN dissection. In standard pelvic LN dissection, lymphadenectomy is confined within obturator fossa, whereas in the extended approach, whose importance has been currently emphasized in many publications, in addition to the boundaries of the standard procedure, the target of lymphadenectomy is extended to include external iliac vein, internal iliac vessels, and femoral canal. In a study conducted by Heidenreich et al., the Authors reported that LN positivity had been $12 \%$ in patients who had undergone standard pelvic LN dissection, but it had raised up to $26 \%$ in the extended procedure. For high-risk patients as those with BNI, extended pelvic LN dissection has been recommended, because, especially in the presence of low-volume, micrometastatic LN involvement, relatively 
longer disease-free survival can be achieved (18). Morbidity of RRP in high-risk PC is similar to that in low-risk patients (4). For these reasons, nowadays RRP in high-risk patients began to be proposed and implemented more frequently. Berglund et al. reported that in patients of high-risk groups who underwent RRP; recovery time, duration of catheterization and continence turnaround time were similar with patients of low risk group (19). In RRP patients with clinical T3, perioperative mortality rates were similar with clinical T2 patients (20). In major centers RRP results of the 5-year and 10year biochemical recurrence free rates were $30-70 \%$ and $15-60 \%$, respectively (21-22). In another study, clinical Tlc-T3b PC patients who underwent RRP or RT were retrospectively compared. At 8-year follow-up results when poor-risk patients treated with RRP were compared to those treated with RT showed that metastatic progression rate of RT group was 9.5\% more than to RRP group (4). One of the most important advantage in RRP is the ability to accurate pathological staging. Approximately $15-25 \%$ of the clinical stage T3 tumors reported as highgraded (23). Consequently RRP may provide more information in determining the need for additional treatment. In addition, LN dissection that was performed during RRP helps us to detect micrometastases which cannot be detected by imaging methods. Otherwise; removal of the seminal vesicles increases the effectiveness of adjuvant treatment (23). In high-risk patients, considering age, general health status and 10 year life expectation, RRP should be recommended as primary treatment. Ten-year biochemical recurrence-free survival rate was $68 \%$ in $35 \%$ of 175 RRP patients who were reported as organconfined disease according to D'Amico risk classification and at the end of follow-up, metastasis-free survival rate and cancer-specific survival rate were $84 \%$ and $92 \%$, respectively (24).

In a retrospective multicenter study, 3828 patients with high risk of PC who underwent RRP were evaluated between years of 1987 and 2010. Ten-year cancer-specific and non-cancer-cause mortality rates were $5.9 \%$ and $14.3 \%$, respectively (25).

In a multicenter European study, were evaluated 1366 patients who were diagnosed as high-risk PC (26). In $37 \%$ of overall patients, organ-confined disease has been identified. This rate in presence of only one of the preoperative risk factor was $45 \%$, but in presence of three risk factors (GS, PSA, clinical stage) organ-confined disease detection rate dropped to $9 \%$.

Fowler et al. reported that the 5-year survival rate of patients with LAPC who were treated with hormone therapy alone was $92 \%$ with a mean follow-up of 78 months (27). Bolla et al. compared the results of combined ADT and RT with those of RT alone in LAPC and reported that overall survival at 5 years was $79 \%$ in the combination group and $62 \%$ in the RT alone group (28). However, patients with PC BNI were generally highgrade (GS 9-10). In the majority of patients short time to progression and lower urinary tract symptoms were observed. We know the high incidence of complications that occurr when salvage surgery performed after RT (29-30). Patients with clinical stage T4 PC are rarely encountered and in only few centers surgical treatment are applied for these patients. Evaluation of this group of patients provides little information about treatment outcomes. Johnstone et al. followed up 1093 clinical stage T4 PC patients at diagnosis and divided them into 5 categories: RRP, only RT, only ADT, RT and ADT combination, and untreated group. The Authors reported that RRP applied for clinical stage T4 PC patients had provided better survival rates when compared with patients who received mono-RT or mono-ADT. Survival rates achieved with surgery were found to be comparable with those obtained with RT and ADT combination therapy (31). Furthermore, the study indicated the survival of RRP over combined RT with ADT for clinical T4 PC with lymph node metastases (32).

When performing cystoprostatectomy (CP) for PC involving the bladder neck, the possibility of overtreatment should be considered. Among clinical T4, compared to patients with PC involving the rectum or pelvic floor muscles, those with BNI may be better candidates for $\mathrm{CP}$ to achieve local cancer control and improvement of quality of life (QOL).

Manifestations of BI consist of hematuria, urinary urgency, pelvic pain, and bladder outlet obstruction. Besides, bladder outlet or ureteral obstruction can lead to renal failure. Many patients might be dependent on lifelong requirement for nephrostomy tubes, ureteral, and urethral stents, and more than one invasive procedure might be needed for routine tube replacements or revision operations. Complications of long-term tube drainage of the urinary tract comprise obstruction, bladder spasms, bleeding, infection, and stone formation. Local symptoms related to BI determine quality of life and reveals the clinical condition. Among adjuvant procedures applied for the management of locally advanced and symptomatic PC patients, systemic treatments as ADT and chemotherapy, local therapies including cryotherapy or surgery (TUR), and local palliative approaches (nephrostomy, and ureteral stent implantation) can be enumerated. The beneficial effect of androgen therapy is restricted with the development of androgen insensitivity. Still, in $89 \%$ of the patients under systemic chemotherapy, local symptoms were maintained (1). Cryotherapy has been tried after failure of RT, but could not prevent lower urinary tract symptoms (33). On the other hand relieve of outlet obstruction by transurethral resection (TUR) is short-lasting and repeated transurethral procedures might be required (34).

Previous reports on patients who had undergone salvage surgery including $\mathrm{CP}$ have demonstrated that they have rarely provided cure. However, in these reports palliative role of CP on patients with local symptoms was not investigated. Leibovici et al. performed palliative $\mathrm{CP}$ on a total of 38 T4 PC patients who developed recurrence following primary disease $(n=17)$, and RT $(n=21)$. The authors compared local symptoms, and the need for surgical intervention for the relief of obstruction before the operation, and at postoperative 3 month follow up during a mean follow-up period of 23 months (35) showing that the role of palliative $\mathrm{CP}$ was not statistically significant. In several institutions, $\mathrm{CP}$ was performed in $\mathrm{PC}$ patients with BNI showing severe LUTS for the purpose of relieving those symptoms. Kumazawa et al. published out- 
comes of CPs they performed on 17 stage T4 patients between the years 1989 and 2005. All the patients in this study, including patients who developed local recurrence, had no local symptoms or no need for catheters for urinary tract obstruction until death. Although no study has compared QOL after conservative treatment and surgical intervention in PC patients with BNI, results indicate that $\mathrm{CP}$ may be a treatment option in these patients. Postoperatively, all patients received additional surgical or medical castration therapy during the mean postoperative period of 89 months. The authors determined 5-year disease-free biochemical survival rate as 62 percent. They demonstrated that palliative $\mathrm{CP}$ can be performed even for lymph node positive patients (36). In the light of these data, we need further researches in order to achieve a more accurate assessment and alternative treatments for LAPC patients.

\section{REFERENCES}

1. Boccon-Gibod L, Bertaccini A, Bono AV, et al. Management of locally advanced prostate cancer: a European consensus. Int J Clin Pract. 2003; 57:187-94.

2. Gallina A, Chun FK, Suardi N, et al. Comparison of stage migration patterns between Europe and the USA: an analysis of 11350 men treated with radical prostatectomy for prostate cancer. BJU Int 2008; 101:1513-1518.

3. Hakenberg O, Frohner M, Wirth M. Treatment of locally advanced prostate cancer the case for radical prostatectomy. Urol Int. 2006; 77:193-199.

4. Eastham JA, Evans CP, Zietman A. What is the optimal management of high risk, clinically localized prostate cancer? Urol Oncol. 2010; 28:557-567.

5. D'Amico AV, Whittington $R$, Malkowicz SB, et al. Biochemical outcome after radical prostatectomy, external beam radiation therapy, or interstitial radiation therapy for clinically localized prostate cancer. JAMA 1998; 280:969-74.

6. Denberg TD, Glodé LM, Steiner JF, et al. Trends and predictors of aggressive therapy for clinical locally advanced prostate carcinoma. BJU Int. 2006; 98:335-40.

7. Meng MV, Elkin EP, Latini DM, et al. Treatment of patients with high risk localized prostate cancer. Results from cancer of the prostate strategic urological research endeavor (CAPSURE). J Urol. 2005; 173:1557-61.

8. Boorjian SA, Thompson RH, Siddiqui S, et al. Long-term outcome after radical prostatectomy for patients with lymph node positive prostate cancer in the prostate specific antigen era. J Urol. 2007; 178:864-870.

9. Zhou M, Reuther AM, Levin HS, et al. Microscopic bladder neck invasion by prostate carcinoma in radical prostatectomy specimens is not a significant independent prognostic factor. Mod Pathol. 2009; 22:385-392

10. Dash A, Sanda MG, Yu M, et al. Prostate cancer involving the bladder neck: recurrence-free survival and implications for AJCC staging modification. American Joint Committee on Cancer. Urology 2002; 60:276-280.

11. Ruano T, Meirelles L, Freitas LL, et al. The significance of microscopic BNI in radical prostatectomies: pT4 disease? Int Urol Nephrol. 2009; 41:71-76.

12. Johnson CW, Anastasiadis AG, McKiernan JM, et al. Prognostic indicators for long term outcome following radical retropubic prostatectomy for prostate cancer involving the seminal vesicles. Urol Oncol. 2004; 22:107-111.

13. Epstein JI, Partin AW, Potter SR, et al. Adenocarcinoma of the prostate invading the seminal vesicle: prognostic stratification based on pathologic parameters. Urology. 2000; 56:283-288.

14. Secin FP, Bianco Jr FJ, Vickers AJ, et al. Cancer-specific survival and predictors of prostate-specific antigen recurrence and survival in patients with seminal vesicle invasion after radical prostatectomy. Cancer. 2006; 106:2369-2375.

15. Masterson TA, Pettus JA, Middleton RG, Stephenson RA. Isolated seminal vesicle invasion imparts beter outcomes after radical retropubic prostatectomy for clinically localized prostate cancer: prognostic stratification of pt3b disease by nodal and magrin status. Urology 2005; 66:152-155.

16. Boorjian SA, Thompson RH, Siddiqui S, et al. Long-term outcome after radical prostatectomy for patients with lymph node positive prostate cancer in the prostate specific antigen era. J Urol. 2007; 178:864-870.

17. Heidenreich A, Varga Z, Von Knobloch R. Extended pelvic lymphadenectomy in patients undergoing radical prostatectomy: high incidence of lymph node metastasis. J Urol. 2002; 167:1681-1686.

18. Berglund RK, Jones JS, Ulchaker JC, et al. Radical prostatectomy as primary treatment modality for locally advanced prostate cancer. a prospective analysis. Urology. 2006; 67:1253-6.

19. Ward JF, Slezak JM, Blute ML, et al. Radical prostatectomy for clinically advanced (cT3) prostate cancer since the advent of prostate specific antigen testing: 15 year outcome. BJU Int. 2005; 95:751-6.

20. Roehl KA, Han M, Ramos CG, et al. Cancer progression and survival rates following anatomical radical retropubic prostatectomy in 3,478 consecutive patients: long-term results. J Urol. 2004; 172:910-4

21. Han M, Partin AW, Pound CR, et al. Long-term biochemical disease-free and cancer- specific survival following anatomic radical retropubic prostatectomy. The 15-year Johns Hopkins experience. Urol Clin North Am. 2001; 28:555-65.

22. Bianco FJ Jr, Scardino PT, Eastham JA. Radical prostatectomy: long-term cancer control and recovery of sexual and urinary function ("trifecta"). Urology 2005; 66:83-94.

23. Kozacioglu Z, Günlüsoy B. Current treatment approaches in locally advanced prostate cancer: urologist approach. J Urooncol Turkey. 2012; 2:124-8.

24. Loeb S, Schaeffer EM, Trock BJ, et al. What are the outcomes of radical prostatectomy for high-risk prostate cancer? Urology. 2010; 76:710-4.

25. Briganti A, Spahn M, Joniau S, et al. Impact of age and comorbidities on long-term survival of patients with high-risk prostate cancer treated with radical prostatectomy: a multi- institutional competing-risks analysis. Eur Urol. 2013; 63:693-701.

26. Briganti A, Joniau S, Gontero P, et al. Identifying the best candidate for radical prostatectomy among patients with high- risk prostate cancer. Eur Urol. 2012; 61:584-592.

27. Fowler JE, Bigler SA, Duncun WL. Hormone therapy for locally advanced prostate cancer. J Urol. 2002; 168:546-549.

28. Bolla M, Gonzalez D, Warde P, et al. Improved survival in patients with locally advanced prostate cancer treated with radiotherapy and goserelin. N Engl J Med. 1997; 337:295-300. 
29. Shekarriz B, Upadhyay J, Pontes JE. Salvage radical prostatectomy. Urol Clin North Am. 2001; 28:545-553.

30. Gheiler EL, Tefilli MV, Tiguert R, et al. Predictors for maximal outcome in patients undergoing salvage surgery for radio-recurrent prostate cancer. Urology. 1998; 51:789-795.

31. Johnstone PA, Ward KC, Goodman M, et al. Radical prostatectomy for clinical T4 prostate cancer. Cancer. 2006; 106:2603-2609.

32. Bolla M1, van Poppel H, Tombal B, et al. European Organisation for Research and Treatment of Cancer, Radiation Oncology and Genito-Urinary Groups Postoperative radiotherapy after radical prostatectomy for high-risk prostate cancer: long-term results of a randomised controlled trial (EORTC trial 22911). Lancet. 2012;380:2018-27.
33. Anastasiadis AG, Sachdev R, Salomon L, Get al. Comparison of health-related quality of life and prostate-associated symptoms after primary and salvage cryotherapy for prostate cancer. J Cancer Res Clin Oncol. 2003; 129:676.

34. Crain DS, Amling CL, Kane CJ. Palliative transurethral prostate resection for bladder outlet obstruction in patients with locally advanced prostate cancer. J Urol. 2004; 171:668.

35. Leibovici D, Kamat AM, Petteway CA, et al. Cystoprostatectomy for effective pallation of symptomatic bladder invasion by prostate cancer J Urol. 2005; 174:6.

36. Kumazawa T, Tsuchiya N, Saito M, et al. Cystoprostatectomy as a treatment of prostate cancer involving the bladder neck. Urol Int. 83:141-5.

\section{Correspondence}

Özgür Haki Yüksel, MD (Corresponding Author)

ozgurhaki@gmail.com

Ayhan Verit, MD. Prof,

Ahmet Ürkmez, MD

Dept. of Urology, Fatih Sultan Mehmet Research \& Training Hospital Içerenköy/Ataşehir Tr- 34752 Istanbul, Turkey 